\title{
1 Electromagnetic Nanonetworks for Sensing and 2 Drug Delivery
}

\author{
3 Renato Iovine ${ }^{1}$, Valeria Loscrì ${ }^{2}$, Sara Pizzi $^{3}$, Richard Tarparelli ${ }^{1}$, and Anna \\ 4 Maria Vegni ${ }^{1}$ \\ $5 \quad{ }^{1}$ Department of Engineering, Roma Tre University, Rome, Italy \\ $6 \quad{ }^{2}$ INRIA, Lille-Nord Europe, France \\ $7 \quad{ }^{3}$ DIIES, Mediterranea University of Reggio Calabria, Reggio Calabria, Italy
}

8

\section{Abstract}

The use of nanodevices for biomedical applications has recently been object of study by researchers. Novel prospectives can be envisaged in the field of nanomedicine, also supported by innovative nanodevices with specific properties.

In this chapter, we present the electromagnetic properties of different metal nanoparticles (i.e., nanocube, nanocylinder, nanorod, bow-tie, biconical nanoparticle, etc.), opportunely functionalized for sensing applications, as well as drugged with medicament to be released to specific locations, for innovative therapeutic treatments. After modeling the design of such nanoparticles, we investigate the channel model adopted in electromagnetic nanonetworks. Basically, we focus on the nanoparticle transmission, diffusion and reception processes, both for extraand in-vivo applications i.e., for the detection of target cells in a biological tissue sample, and for drug delivery via nanoparticle adsorption, respectively. Numerical results obtained through full-wave simulations have shown the effectiveness of electromagnetic nanoparticles for specific biomedical applications (e.g., DNA alteration detection). Finally, we highlight that in this chapter the electromagnetic properties that are described are used for sensing and drug delivery, and not for communication among nanoparticles.

\section{Introduction}

The concept of nanomedicine arises from the visionary idea that miniaturized devices at nanoscale level (i.e., nanodevices or nanorobots) could be designed, manufactured, and introduced into the human body, for therapeutic aims (e.g., cellular repairs at the molecular level) [1]. The possibility of applying nanotechnology to medicine has been object under study for the last decades, and it represents a 
new approach based on the comprehension and deep knowledge of the properties of the matter at the nanoscale level [2].

The intrinsic behavior and characteristics of nanodevices distinguish them from traditional devices working at the macroscale level, and particular features at the nanoscale level should be addressed [3]. Indeed, the properties of the matter drastically change, making it necessary a synergy among several different disciplines, in order to define novel communications techniques and design efficient nanodevices [4].

Generally, nanodevices represent the most basic functional unit with passive features, which allow performing very easy tasks, like sensing or actuation. A set of nanodevices, sharing the same medium (e.g., the biological tissue or the blood flow) and performing multiple tasks, form a nanonetwork [5]. Nanonetworks allow to expand the number and range of applications envisioned for single nanodevices, since collaborative tasks can be done by different nanodevices. Nowadays, applications are foreseen in four main fields, namely (i) biomedical, (ii) environmental, (iii) industrial and consumer goods, and (iv) military and defense $[3,6]$.

Communication and signal transmission techniques to be utilized/used in nanonetworks are one of the most challenging topics, due to the limited computation skills of single nanodevices. Classical communication and network paradigms cannot be directly utilized in nanonetworks, since the poor capabilities of nanodevices pose novel challenges, establish new requirements and show novel properties that need to be opportunely addressed. As an instance, current encoding and decoding techniques are not feasible due to very limited processing capability of nanodevices, as well as traditional transceiver circuitries cannot be mounted into them due to the limitation of the nanoscale. Also novel mobility models should be addressed accordingly to this particular field, due to specific physical rules in this regime [7].

The biomedical field is one of the most challenging area of application of nanonetworks, as well as the most intriguing due to a variety of biomedical scenarios. Indeed, in the biomedical field, nanonetworks are expected to provide a perfect interface to interact with single molecules, proteins, DNA sequences and the major components of cells. Both in-vivo and extra-vivo applications of biocompatible nanodevices are largely investigated. As an instance, the use of nanosensors to detect chemical compounds in concentrations, or the presence of different infectious agents, such as virus or bacteria is an objective of several research studies $[8,9]$.

In the biomedical field, we highlight three main applications i.e., (i) health monitoring systems, (ii) Drug Delivery Systems (DDS), and (iii) bio-hybrid implants. In health monitoring systems, the use of nanosensors allows detecting and monitoring different levels of molecule concentration in the blood (e.g., sodium, glucose and other ions), as well as the presence of infectious intra-body agents. The DDS use nanoactuators capable of releasing nanoparticles, drugs or biomolecules in specific locations of the body; this means that drug molecules are released 
1 locally and are adsorbed only by the diseased cellular membranes, so that patients 2 benefit from a less invasive and much more efficient treatment. Finally, bio-hybrid 3 implants rely on nanodevices able to cooperate not only with each others, but also 4 with biological components (e.g., to restore the central nervous tracks or to sup5 port the immune system).

As the design and manufacturing of devices at the nanoscale advance, new possibilities are given for the interconnection among nanodevices and new challenges rise in the development of protocols and channel models for nanonetworks. Based on the different types of nanodevices (i.e., biological, and electromagnetic), nanonetworks are mainly classified as (i) molecular [10], and (ii) electromagnetic [3].

In this chapter, we investigate electromagnetic (EM) nanonetworks properties with different nanoparticles, working in the $\mathrm{THz}$ band, used for sensing and drug delivery, and foreseeing the transmission and reception of electromagnetic radiation from components based on nanomaterials. In [3], Akyildiz and Jornet present the architecture of a nanosensor device as comprised of nanosensors, nanoactuator, nano-memory, nano-antenna, nano-EM transceiver, nano-processor and nanopower unit. All these components allow the integrated device to sense, compute or even perform local actuation. Furthermore, the authors foresee that nanosensor devices will potentially communicate among them in the terahertz band (i.e., 0.1$10.0 \mathrm{THz}$ ).

The main challenges of electromagnetic-based nanonetworks are expressed in terms of THz channel modeling, information encoding and protocols for nanosensor networks. A physical channel model for wireless communication in the $\mathrm{THz}$ band has been developed by Jornet and Akyildiz in [11]. The presented model computes the signal path loss, the molecular absorption noise and, ultimately, the channel capacity of EM nanonetworks. In [12], a modulation and channel sharing mechanism based on the asynchronous exchange of femtosecond-long pulses transmitted through an on-off keying modulation is proposed for the transmission of binary streams among nanodevices of an EM nanonetwork. A medium access control protocol for EM nanonetworks built on the top of the pulse-based communication scheme in [12] for the coordination of multiple simultaneous transmissions is presented in [13]. The proposed protocol is tailored to the peculiarities of the terahertz band and is constituted by two main stages i.e., $(i)$ the handshaking process, and (ii) the transmission process. Finally, in [14] Jornet and Akyildiz have developed an energy model for self-powered nanosensor motes, which successfully captures the correlation between the energy harvesting and the energy consumption processes. The energy harvesting process is realized by means of a piezoelectric nanogenerator, for which a new circuital model is developed that can accurately reproduce existing experimental data. The energy consumption process is due to the communication among nanosensor motes in the $\mathrm{THz}$ band.

The application of nanoparticles for combined targeting and delivery of diagnostic and therapeutic agents has received significant attention in the last years [15]. Colloidal metallic nanoparticles have been recently investigated in the field 
of nanomedicine, especially for drug delivery systems [16]. Noble metals, like silver and gold, are largely used for the design of nanoparticles, in the field of sensing applications, as well as acting as carriers of medicament molecules. Particularly, colloidal silver has been used as an antibacterial agent by weakening DNA replication and inactivating proteins, while gold has low toxicity to biological systems, and so results inefficient for antibiotic therapy [17]. In addition, nanoparticles are also engineered to provide sustained drug release [18] [19], especially beneficial for chronic therapies. In [20], the authors use gold nanoparticles for diagnostic and drug delivery applications by exploiting chitosan. The use of chitosan serves dual purpose by acting as a reducing agent in the synthesis of gold nanoparticles, and also promotes the penetration and uptake of peptide hormone insulin across the mucosa. As a first step towards real implementable solutions, in [21] nanomachines have been designed for medical applications to colonize and autonomously work inside the human body.

Models for particulate DDS, based on the injection of drug molecules, have been proposed in [22, 23]. In [22], the authors consider the human blood vessels to model the medium where molecules diffuse, subjected to the cardiac input. Drug molecules are then allowed to move in every location of the cardiovascular system. On the other side, the possibility of using a swarm of bio-nanomachines (i.e., nanoscale devices composed of natural or synthetic biological materials) with collective behaviour, in order to perform collaborative tasks like target detection and molecule guiding, has been investigated by Nakano et al. in [23]. The same concept of swarm of nanomachines has been utilized in [24], where endogenous diseases of the brain are treated by means of nanodevices that communicate through acoustic signals. Finally, a multi-source nanonetwork model for extra-vivo biomedical diagnosis applications, specifically the detection of DNA alterations, is presented in [25].

In this chapter we focus on the use of electromagnetic nanodevices for biomedical applications, specifically sensing of DNA alterations and drug delivery through oral ingestion. After describing the main features of electromagnetic nanoparticles used in biomedical applications, we consider a physical end-to-end model, and investigate how nanoparticles are transmitted, diffuse, and finally are captured. Furthermore, we also present simulation results that show the capability of nanosensors to operate in biomedical applications.

This book chapter is organized as follows. In Section 2, we present different types of electromagnetic nanodevices (i.e., nanoparticles), and describe their specific features and properties. All these nanoparticles represent simple nanonodes, comprising an electromagnetic nanonetwork, and are then subjected to specific processes (i.e., transmission, diffusion, and reception). These are then investigated in Section 3. The sensing and drug delivery applications of nanoparticles are finally presented in Section 4; we show specific use cases, by means of simulation results. Finally, conclusions and considerations on future investigations are drawn at the end of the chapter. 


\section{Electromagnetic nanoparticles for biomedical applications}

In the last few years the fabrication of nanostructures received too much attention. Optical properties of metallic nanoparticles make them suitable for biomedical applications [26]. In particular, gold nanoparticles have inner electromagnetic properties, depending on the size, shape, geometrical parameters, and the surrounding dielectric environment Refractive Index (i.e., $R I$ ). To be more precise, the strongly enhanced Localized Surface Plasmon Resonance (LSPR) of this metal, at optical frequencies, makes it good light scatters and absorbers [27]. In addition to this, gold nanoparticles offer good bio-compatibility, optimal synthesis and conjugation properties [28], and are useful tools as contrast agents in cellular and biological imaging [29].

Nanoparticles are of great interest in biomedical applications such as light scattering microscopy-based imaging, sensing applications, and photothermal therapy for superficial and deep tumors treatment [30]. New sensing techniques to reveal malignant tissues are needed. For many optical sensors, proposed in literature, the presence of the biological sample is detected by measuring its refractive index in several ways [31].

One of the most used techniques for biosensing is based on the LSPR phenomenon [32], which occurs when an electromagnetic plane wave impinges on metallic nanoparticles that are electrically small. In this condition the free electrons of the nanoparticle follow collectively the electromagnetic oscillations. This phenomenon derives from the peculiar metallic nanoparticles optical properties, and leads to a strong local electromagnetic field enhancement. Therefore, the resonant frequency of the electron motion strongly depends on the nanoparticle size, shape, composition, and surrounding dielectric environment [33].

In order to explain the nanoparticle electromagnetic properties, in terms of scattering and absorption cross-section, the following assumptions need to be established:

- The particle size is much smaller than the wavelength in the surrounding medium. In this case, under the limit of electrically small particles, the electromagnetic field is approximately constant over the particle volume, and then the resonant behavior of the structure can be studied in terms of a quasi-static approximation;

- The considered particle is homogeneous and isotropic. In addition, the surrounding material is a homogeneous, isotropic and non-absorbing medium.

Under previous assumptions, it is possible to relate the geometrical properties of the structure to its electromagnetic ones (i.e., scattering and absorption), by developing its polarizability analytical expression: 




(a)

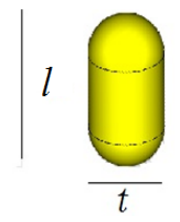

(b)

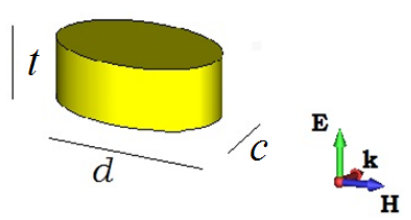

(c)

Figure 1. Geometrical sketch of the nanostructures: $(a)$ cube, $(b)$ rod, and $(c)$ elliptical cylinder.

$5 \quad \underline{\underline{\alpha}}=V \varepsilon_{e} \sum_{n=1}^{3} \frac{\varepsilon_{i}-\varepsilon_{e}}{\varepsilon_{e}+L_{n}\left(\varepsilon_{i}-\varepsilon_{e}\right)} \underline{u}_{n} \underline{u}_{n}$

where $V$ is the nanoparticle volume, $\varepsilon_{e}$ is the dielectric permittivity of the surrounding environment, $\varepsilon_{i}$ is the complex dielectric permittivity of the metallic nanoparticle, $\underline{u}_{n}$ are unit vectors in the direction of the principal axes of the nanoparticle, and $L_{n}$ (with $n=[1,2,3]$ ) are the three components of the corresponding depolarization dyadic, that is

$\underline{\underline{L}}=L_{1} \underline{u}_{1} \underline{u}_{1}+L_{2} \underline{u}_{2} \underline{u}_{2}+L_{3} \underline{u}_{3} \underline{u}_{3}$.

The depolarization dyadic $L$ is an essential concept in the evaluation of the electric field in the source region, in order to establish the electromagnetic response of an arbitrary shape of nanoparticle through the polarizability expression. From a physical point of view, it is a dimensionless matrix that allows taking into account anisotropic nanoparticles.

Following the same procedure in [34], it is possible to develop new depolarization factors for specific nanoparticles, and then new analytical closed-form formulas for scattering and absorption cross-section can be derived.

The general expression describing the extinction cross-section properties for different nanoparticles shapes can be written as the sum of absorption and scattering phenomenon as follows:

$C_{e x t}=k \cdot \operatorname{Im}[\alpha]+\frac{k^{4}}{6 \pi}|\alpha|^{2}$

where $k=\frac{2 \pi n}{\lambda}$ is the wavenumber, $\lambda$ is the wavelength, and $n=\sqrt{\varepsilon_{e}}$ is the refractive index of the surrounding dielectric environment. The extinction cross-section represents the effective area that governs the probability of scattering and absorption event by a nanoparticle. In general, the extinction cross-section is different from the geometrical cross-section of a particle, and it depends upon the wavelength of light and the permittivity, shape and size of the particle. In terms of area, 
the extinction cross-section $\left[\mathrm{nm}^{2}\right]$ is the sum of the cross-sections due to absorption and scattering.

Following this way, it is possible to predict in accurate manner the electromagnetic response of each nanoparticle shape. This aspect is crucial in project phase, and allows optimizing the nanoparticles sensibility for specific applications.

As shown in [35], considering cube, rod and elliptical cylinder nanoparticles, for each of the considered structures the analytical models have been calculated. By assuming that the structures are excited by a plane wave, having the electric field $\mathbf{E}$ and the vector propagation $\mathbf{k}$ directed as depicted in Figure 1, the polarizability of cube, rod and elliptical cylinder nanoparticles follow, respectively:

$L_{\text {cube }}=10 \sqrt{2}\left(1-\frac{1}{\sqrt{2}}\right) \pi \cdot \frac{1}{l}$,

$$
L_{\text {nanorod }}=1-\frac{1}{\sqrt{1+\left(\frac{t}{2}\right)^{2} \cdot \frac{1}{\left(\frac{t}{2}+\frac{l}{4}\right)^{2}}}},
$$

$L_{\text {elliptical cylinder }}=\frac{1}{\pi}\left(1-\frac{t}{4 \sqrt{\frac{t^{2}}{2}+\frac{t^{2}}{16}}}\right) \cdot E\left[\frac{1}{1-\left[1-\left(\frac{c}{2}\right)^{2} \cdot\left(\frac{2}{t}\right)^{2}\right]}-1\right]$

where $E$ [ ] is the complete Elliptic Integral of the second kind, $l[\mathrm{~nm}]$ is the cube side length, as well as the height of the nanorod and of the elliptical cylinder, $d$ $[\mathrm{nm}]$ and $c[\mathrm{~nm}]$ are the elliptical cylinder base axes lengths, $t[\mathrm{~nm}]$ is the rod thickness, as well as the length of elliptical cylinder.

Replacing (4), (5) and (6) in (1), and later in (3), the extinction cross-section spectrum for cube, rod and elliptical cylinder nanoparticles can be obtained [35]. In this way it is possible to predict the optimal geometrical parameters, in order to optimize the nanostructure for specific applications (i.e., biosensing applications). For example, in [36], a sensor revealing tumor and adipose tissue is presented. It consists of a gold linear chain of four nanocubes deposited on a silica substrate, and allows revealing the rat mammary cancer and adipose tissue by LSPR shift, as shown in Figure 2. 


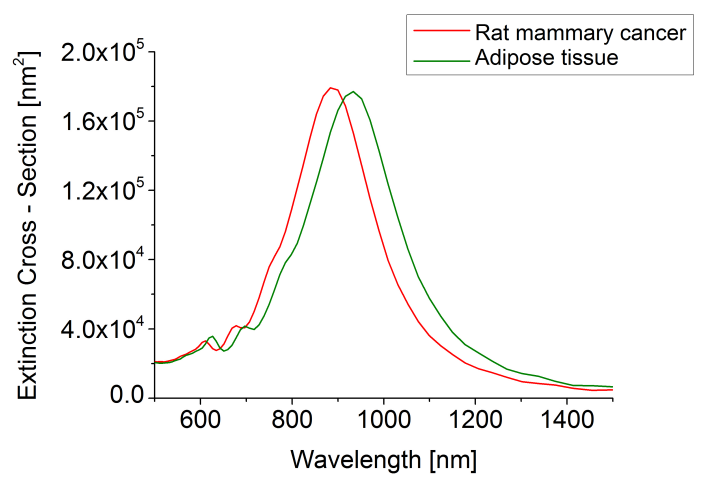

Figure 2. Extinction Cross-Section spectra for rat mammary cancer $(R I=1.39)$, and adipose tissue $(R I=1.467),[36] . R I$ values are known in literature.

By using very small inter-particle distance among the nanoparticles it is possible to obtain high-scattering and low-absorption efficiencies. These properties are very important for biosensing applications. In fact, high-absorption efficiency could heat the biological sample invalidating medical diagnosis. The biological sample used to test this device is an in-silico replica with values of $R I$ taken from the literature. In particular the $R I$ values of rat mammary adipose and tumor tissue have been considered.

Finally, by using the same physical principle, in [37], a label-free immunosensor was designed and fabricated for sensitive of alpha-fetoprotein (AFP) of gold nanorods.

In literature various shapes of metallic nanoparticles are used for biosensing applications. For example, in [38] the ellipsoidal gold nanoparticles have been analyzed, and a new analytical study of metallic nanoparticles working in the infrared and visible frequency range has been presented. The approach proposed in [38] is a useful tool to design nanostructures for sensing applications.

Recently, another important property of metallic nanoparticles has been analyzed and exploited. In [39], an analytical and numerical investigation for modified gold nanorods, operating in the visible and in the infrared regime, is proposed. The modified particle consists in a core/shell structure (i.e., silica core, and gold shell) embedded in a dielectric environment, as shown in Figure 3. In order to study and to tune the electromagnetic nanostructure, a new analytical model has been developed. The electric field distribution at the resonant wavelength demonstrates the intensity of the electromagnetic field in the neighborhood nanoparticle, as shown in Figure 4. This result derives from the silica core that allows the field intensification.

Exploiting the obtained model, the nanoparticle sensitivity was studied and verified by full-wave simulations. In particular, being an asymmetric structure, the electromagnetic properties, in terms of extinction cross section (i.e., absorption and scattering) for both longitudinal and transverse modes excitation, have been evaluated. 


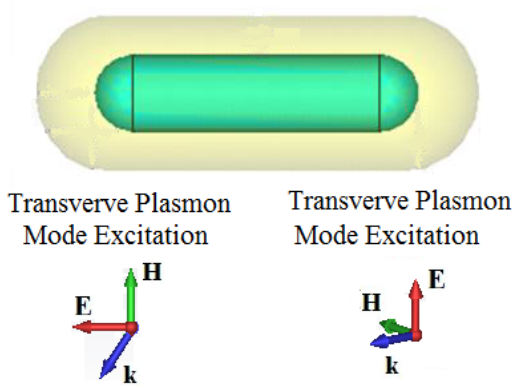

Figure 3. Core/shell nanorod particles, and two mode excitations.

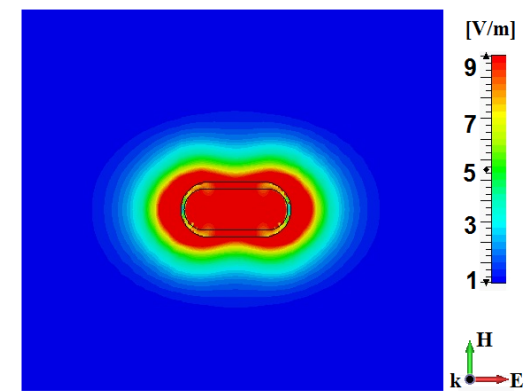

Figure 4. Near electric field distribution at the resonant wavelength (i.e., $696 \mathrm{~nm}$ ) of nanorod.

As explained in [39], the difference between the longitudinal and transverse sensitivity can be explained in the following manner: it is known that nanoshells possess two resonances arising from the interaction between the classical plasmon resonance of the solid particle, and the resonance of the dielectric core inside the metal. The particle plasmon resonance holds increased sensitivity to the dielectric environment variation, especially for the longitudinal polarization. Instead, the dielectric cavity resonance is much more sensitive to changes in dielectric properties within the nanoparticle core and shell dimensions for transverse polarization.

Modified nanorods represent useful tools for sensing, since they combine two main optical properties of both nanorods (i.e., the high Aspect Ratio), and nanoshells (i.e., core/shell thickness), in order to reach the higher sensitivity. Thus, it allows additional degrees of freedom for the optical tenability of such particles.

In the last few years several research have focused the attention to metallic nanoparticles in a coupled configuration. In fact, to arrange gold nanoparticles in this way allows obtaining a greater intensification of the electromagnetic field, with respect to the single-element configuration. This aspect provides a nanostructure with major sensitivity in terms of LSPR shift [40]. Because the resonance of this coupled mode is sensitive to the gap distance change in the order of few tens of nanometers or less, it is inversely possible to measure such distances by monitoring the scattering of the particle pair, the so-called plasmon ruler, as well described in [40]. 


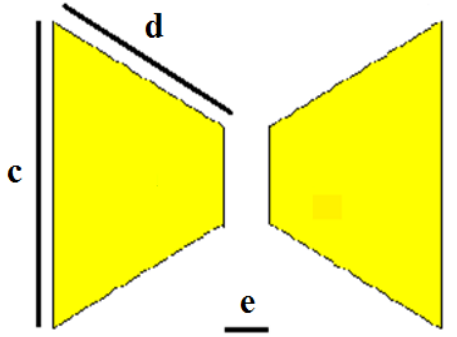

(a)

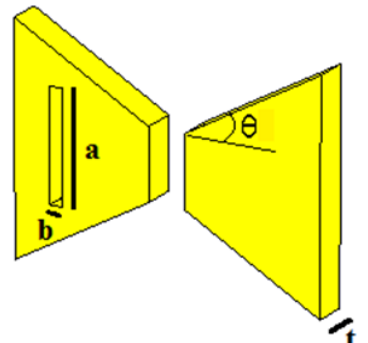

(b)

Figure 5. Geometrical sketch of the bow-tie nanoparticle. (a) Top view of classical bow-tie particle, and $(b)$ perspective view of modified bow-tie nanoparticle by dielectric incision. Geometrical parameters: $a=80 \mathrm{~nm}, b=10 \mathrm{~nm}, c=160 \mathrm{~nm}, d=155 \mathrm{~nm}, e=20 \mathrm{~nm} \theta=30^{\circ}$, and $t=25 \mathrm{~nm}$.

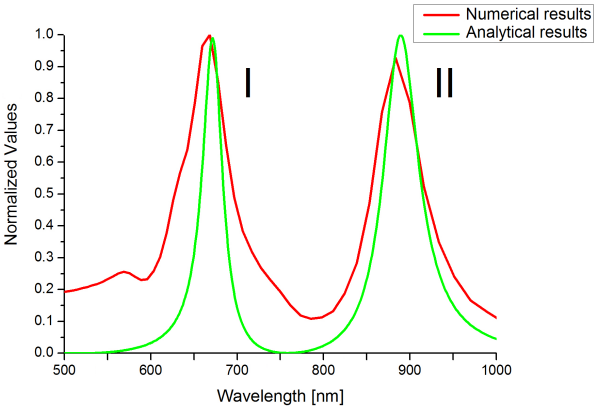

(a)

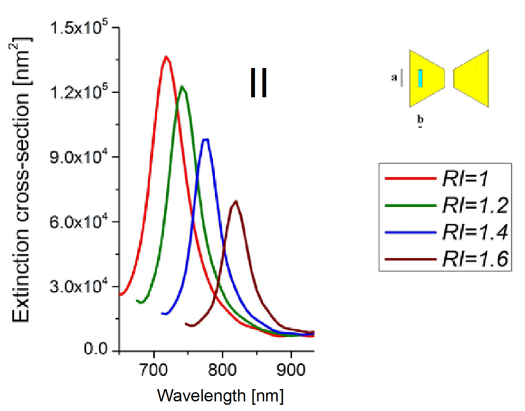

(b)

Figure 6. Extinction cross-section spectra for modified gold bow-tie nanoparticles, in the case of (a) analytical-numerical model comparison (normalized values) [41], and (b) local RI variation, inside the teal blue area.

Another property of metallic nanoparticles consists of multi-resonant approach. In [41], a multi-resonant bow-tie structure is presented. The classical bow-tie nanoparticle consists of two opposing truncated gold prisms, as depicted in Figure 5 (a). In the classical bow-tie particle, the reason for the employment of the dielectric hole, as shown in Figure $5(b)$, is the possibility to excite a new resonant frequency on the same structure in order to achieve a multi-band behavior.

In this configuration the particles exhibit an additional resonant frequency, as reported in the following Figure 6. In order to tune and control the physical phenomenon, and to design it with specific requirements, in [41] the analytical model has been developed. A good agreement among numerical and analytical results was achieved, as shown in Figure $6(a)$. We notice that the nanostructures have been analyzed in terms of sensitivity properties, and results reveal that the modified bow-tie structure can be applied for biomedical applications.

For example, it is well known that in the VIS-NIR tissues have a specific $R I$ value, representing a unique physical property. Local $R I$ changes in the tissue are related to different pathological conditions. In disease states, such as neoplasm or inflammation, color of tissue changes due to the change in $R I$, which in turn is re- 
1 lated to the relative permittivity of the tissue. Therefore, the dielectric constant 2 changes as a result of different re-distributions in electron density of the tissue and 3 it can be associated to different pathological conditions.

The obtained results are shown in Figure $6(b)$, where the local $R I$ variation causes a selective shift in the extinction cross-section spectra (Peak II). In particular, Peak II associated to the local $R I$ variation inside the teal blue area shifts from $717 \mathrm{~nm}$ to $820 \mathrm{~nm}$ for $1<R I<1.6$. The mean sensitivity value is $171 \mathrm{~nm} / R I U$.

\section{Transmission, Diffusion and Reception process in}

\section{Electromagnetic Nanonetworks}

10 After describing the main features of electromagnetic nanoparticles used in biomedical applications, like sensing and drug delivery, in this section we investigate how nanoparticles are transmitted, diffuse, and finally are captured in a physical end-to-end model.

The concept of electromagnetic nanonetworks holds classical basics of both information theory, and electromagnetic propagation. The specific feature is the use of engineered metallic nanoparticles, working in the $\mathrm{THz}$ frequency range, which are exploited by means of the use of LSPR phenomenon.

From the communication and channel modelling point of view, the electromagnetic nanonetworks represent a nanonetwork where the nanonodes are metallic nanoparticles, as small as molecules, and then depending on diffusion-based nanocommunications. Following this consideration, we can compare a flow of nanoparticles as a flow of molecules, and the electromagnetic theory laws are applied for sensing applications, by exploiting the interaction of an impinging electromagnetic wave with the metallic surface of the nanostructures.

In a nanonetwork utilized for sensing or drug delivery purposes, from the point of view of the information theory, the transmitter is represented by the source (i.e., nanomachine) that emits nanoparticles, while the receiver is the set of target cells laying in the area where a phenomenon needs to be sensed or a drug needs to be delivered. Due to the particular nature of nanoparticles (i.e., very small devices at nanoscale level), the propagation process allows nanoparticles to move along the space linking the transmitter to the receiver, according to a diffusion model.

Communications via Diffusions (CvD) arise from molecular nanonetworks [42, 60], where specific molecules, called messenger molecules, act as the information carriers between two nanomachines residing in close-to-medium proximity to each other in a fluid environment. 


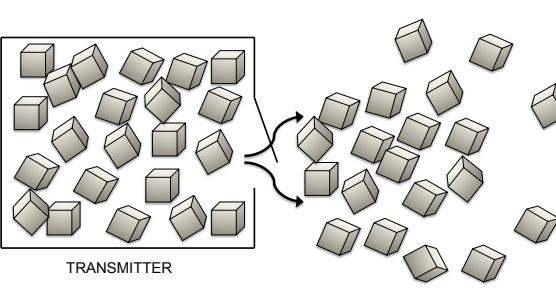

$\nabla$

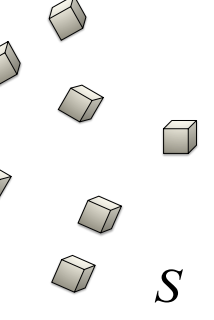

$S$

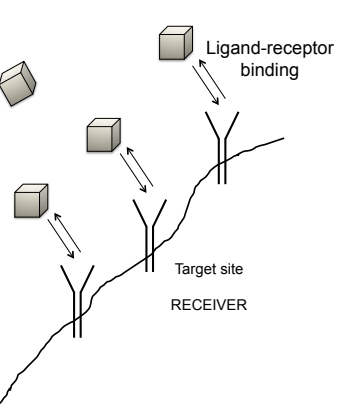

Figure 7. End-to-end physical model of an electromagnetic nanonetwork for sensing or drug delivery purposes. The transmitter is represented by a nanomachine, filled with a nanoparticle concentration, exiting and diffusing into the medium (i.e., the space $S$ ). The nanoparticles act either as drug carriers for drug delivery applications, or as bio-functionalized nanodevices for sensing applications. The receiver is often represented by a target site (e.g., a group of tumor cells), with receptors for capturing the nanoparticles. The sensing and drug delivery applications occur only when the nanoparticles have been bound to the receiver's receptors.

In our vision, $\mathrm{CvD}$ systems can be also applied to electromagnetic nanonetworks, due to common features between molecules and metallic nanoparticles. Indeed, a single nanoparticle is an indivisible object, like a molecule, which is released (during the emission process), or collected by means of chemical reactions (during the reception process). At the same time, a nanoparticle can act as messenger nanoparticle, since it can carry information, such as drug concentration. Furthermore, metallic nanoparticles constituting the electromagnetic nanonetwork are passive nanodevices that is, they cannot transmit data information (e.g., drug molecules) by themselves, but need to be impinged by an electromagnetic wave in order to release information. Due to all these features, electromagnetic nanoparticles are assumed to be very similar to molecules.

Several works have thought of the transmitter as a nanomachine or a bioengineered cell capable of emitting nanoparticles and releasing them into the medium to change their concentration [43]. Similarly, the receiver is capable of capturing the nanoparticles, by using ligand-receptor bindings [44], [45].

If a nanoparticle collides with a receiver, it means that the nanoparticle hits the receiver, and the nanoparticle is then removed from the system since the couple ligand-receptor at a receiver forms a chemical bond with the messenger nanoparticle [46]. Indeed, it is assumed that the whole surface of the receiver is composed of receptors, which are able to bind with the messenger nanoparticles. It follows that each received nanoparticle constitutes the signal just once. This process is named first hitting process [67]. On the other side, if a nanoparticle hits a transmitter, it bounces back from the transmitter since a transmitter does not have the same ligand receptors on their outer shell. 
The receiver is often a biological sample (i.e., a tumor tissue), and has a large number of binding places, so that it can estimate the concentration by averaging over all the created bonds.

From all previous considerations, a nanonetwork can be represented by the following main processes $[6,42]$ :

1. Emission: this process investigates how nanoparticles are transmitted from a nanomachine (or a set of nanomachines);

2. Diffusion: illustrates how nanoparticles diffuse along the gap that separates the transmitter from the receiver lying in the common space $S$;

3. Reception: describes how nanoparticles are captured by the receiver, by means of ligand-receptor bindings.

The transmitter releases a number of nanoparticles in a time slotted fashion. These messenger nanoparticles scatter in the medium following the probabilistic diffusion dynamics in the environment. Some of these released nanoparticles are received via receptors in the cell membrane.

All the above-mentioned processes take place inside a space $S$, which is strictly related to the application for which the nanonetwork is designed, and it is initially filled with a homogeneous concentration of particles equal to zero. The physical end-to-end model is depicted through the scheme in Figure 7, where the main modules of emission, diffusion and reception of nanoparticles are represented.

In the recent years, many studies have focused on the channel capacity and propagation dynamics of the CvD medium [46, 48, 49, 50]. Some of these propagation process studies consider the probabilistic behaviour of the channel as the transfer function of the system, while others model it as a unique noise source inherent to a diffusion medium. According to the aforementioned studies on channel capacity, it has been shown that the reliability of the transmission diminishes exponentially with increasing transmission range, while the average end-to-end delay increases exponentially. These results limit the effective communication range of the CvD systems to a few tens of micrometers.

Several studies on CvD systems focus on a single transmitter single receiver systems. However, when there are more communicating couples in the environment, additional issues arise (i.e., interference and noise). An important issue is the interference between closely placed transmitting couples in the same medium. When two or more transmitting pairs try to communicate simultaneously using the same technique and the same type of messenger nanoparticles, their signals affect each other and reduce/increase the Signal-to-Noise and Interference Ratio (SINR) of all nearby transmissions. 


\section{Emission process}

The emission process aims to modulate the nanoparticle concentration rate (i.e., $\left.r_{T}(t)\right)$ at the transmitter. This is the first process occurring in the end-to-end physical model, as depicted in Figure 7.

In [49], Pierobon and Akyildiz developed a mathematical framework to interpret the diffusion-based nanoparticle communications, in the simple case of one transmitter and one receiver. The emission process has been modelled by means of the electrical circuit theory, considering a RC circuit, where the capacitor charging/discharging current is related to the net flux of nanoparticles. Then, the particle concentration rate $r_{T}(t)$ can be expressed as:

$r_{T}(t)=\frac{d c(t)}{d t}$.

The approach in [49] represents the classical representation of nanoparticle emission in diffusion-based nanonetworks. Following this vision, the work in [50] is based on a pulse-based modulation scheme applied to diffusion-based communication nanonetworks. Whenever a transmitter (i.e., a nanomachine) wants to communicate some information to its neighbours, it instantaneously releases a pulse of nanoparticles (e.g., molecules). This creates a spike in the nanoparticle concentration at the transmitter location, which then propagates through space and time. Notice that the nanoparticle concentration does not only depend on time, but it also varies along the space. The propagation of this pulse can be analytically modelled by solving Fick's Laws of Diffusion.

If the transmitter releases $Q$ molecules at the instant $t=0$, the molecular concentration at any position $x[\mathrm{~nm}]$ in space, from the transmitter location is given by:

$c(x, t)=\frac{Q}{(4 \pi D t)^{3 / 2}} e^{-x^{2} / 4 D t}$,

where $t$ [s] is the time, and $D\left[\mathrm{~cm}^{2} / \mathrm{s}\right]$ is the diffusion coefficient, assumed as a constant value for a given fluidic medium, and depending on the size and shape of the nanoparticles, as well as the interaction with the solvent and viscosity of the solvent. Eq. (9) allows obtaining the concentration measured by a receiver located at a distance $x=r[\mathrm{~nm}]$ from the transmitter as a function of time. We can observe that the concentration initially measured by the receiver is zero, but it sharply increases until reaching its maximum. The time instant at which this maximum occurs can be interpreted as the pulse delay. After the concentration peak is reached, the impulse response slowly decreases, forming a long tail due to the effect of diffusion. In 1-D environments, it is easy to obtain the closed form solution for the 
1 first hitting probability function, since the nanoparticles diffusing along the fluid 2 hit the receiver with probability 1 , thus representing a recurrent process. The ex3 pression of the first hitting probability $F_{h}\left(r_{0}, t\right)$ for a point source in 1-D environ4 ment is

$5 \quad F_{h}\left(r_{0}, t\right)=\frac{r_{0}}{\sqrt{4 \pi D t^{3}}} e^{-r_{0}^{2} / 4 D t}$,

6 where $r_{0}[\mathrm{~nm}]$ is the distance to the receiver. On the other side, in 3-D environ7 ments solving the first hitting probability function is a hard surface integration or 8 differential equation problem, since there is a nonzero probability for a diffusing 9 nanoparticle to miss the receiver [66]. As a solution for the 3-D case, Yilmaz et al. 10 [67] simulate the first hitting process following a Gaussian distribution for each 11 movement at each dimension in one time step made by messenger molecules, i.e.

$\Delta x_{i} \simeq \mathcal{N}(0,2 D \Delta t)$

where $\Delta x_{i}[\mathrm{~nm}]$ is the displacement at the $i$-th dimension (with $i=\{1,2, \ldots, n\}$ ), $\Delta t[\mathrm{~ns}]$ is the time step, and assuming a reflection or removal of those particles that hit to transmitter or receiver, respectively.

In some biomedical applications, like extra-vivo sensing, a multi-source nanoparticle emission process can be required. As an instance, target cell detecting can be tailored to the detection of DNA alterations of the BReast CAncer susceptibility gene 1 (BRCA1). As known, BRCA1 is a human gene belonging to a class of genes known as tumor suppressors. Mutation of these gene causes a genetic susceptibility to breast cancer, and changes in its alternative splicing profile have been associated with malignant transformations that greatly increase woman's risk of developing breast cancer $[51,52]$.

In this scenario, the emission process should be designed to allow the capture of target BRCA1 DNA sequences through a set of unit cells, each of them composed of square gold patches (receptors) deposited on a silica substrate. Each receptor is functionalized with the structure of the BRCA1 splice with the corresponding sandwich assays. The chemical receptors are BRCA1 alternative splice variants i.e., $\Delta(5 q, 6), \Delta(9,10)$, and $\Delta(11 q)$ [53]. Figure $8(a)$ depicts the end-toend physical model for the detection of BRCA1 DNA alterations [25].

In this case, each nanomachine can emit a particular type of nanoparticles (i.e., with a given shape and size), and the chemical receptors are accordingly functionalized for the capture of one type of nanoparticle. Then, the transmitter (i.e., represented by the syringe) is comprised of three nanoparticles flows, injected all together in a sink with a biological tissue, and each constituted of different shapes nanoparticles (see Figure $8(a)$ ). The receiver is represented by the unit cell of a sensing platform, as described in the next section. 


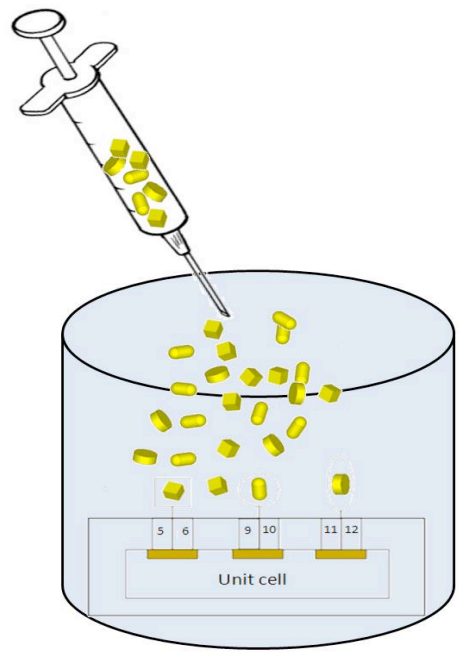

(a)

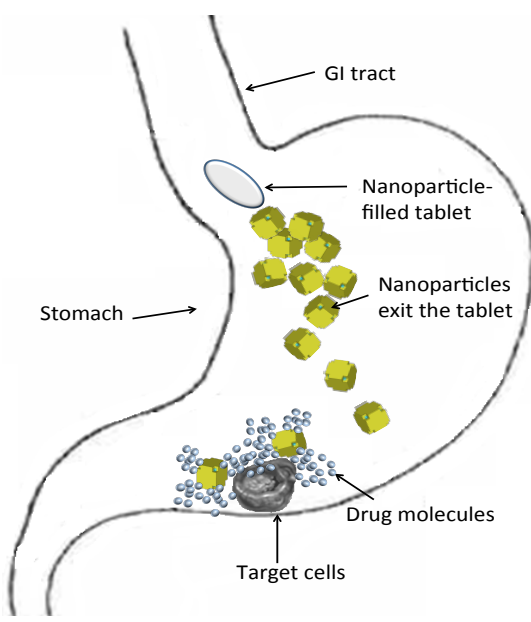

(b)

Figure 8. End-to-End physical model in (a) sensing (i.e., multiple-detection of DNA alterations) [25], and (b) drug delivery (i.e., stomach disease therapy) applications.

A multi-source nanonetwork can suffer from synchronous and asynchronous transmissions, which can degrade network performance with an increase of interference. The same consideration exists at the receiver side, where a selective reception of nanoparticles occurs. As expected, a nanoparticle (a)synchronous transmission corresponds to a (a)synchronous reception. In the case of asynchronous emission, just one nanomachine has transmitted a flow of nanoparticles, and then just one kind of ligand-receptor bind can occur (e.g., the nanomachine 2 has transmitted a flow of nanocubes), while in the synchronous case, all the nanomachines have transmitted the own nanoparticles.

In the case of synchronous emission, nanoparticles detection issues are limited and not likely to occur since each nanoparticle is allowed forming a ligandreceptor binding with a given receptor. As a result, no ambiguity issue regarding the nanoparticle detection can occur. However, during the diffusion process, the flows of nanoparticles can be affected by interferences (i.e., other nanoparticles can be recognized as belonging to the same flow), and this provides a change in the emission rate of the single nanomachine.

Finally, in oral drug delivery systems, the drug delivery process can occur via different modalities, such as oral ingestion, and injection.

In [22], the diffusion of nanoparticles within the human body is modelled as a diffusion-based nanonetwork, under the specific features of the blood flow.

In oral drug delivery, the recommended total dose of drug is delivered through the medicament concentration filled into a sachet, and encapsulated or compressed into a tablet, as well as in a liquid fluid. Spatially controlled drug delivery can be 
obtained by conjugating drug-encapsulated nanoparticles with targeting ligands, which could facilitate the preferential delivery of nanotherapeutics to the sites of interest, while reducing undesired side effects elsewhere. In this case, metallic nanoparticles, filled with typical drugs for antitumoral therapy, and covered with a polymer that allows a higher resistance to the Gastro-Intestinal tract barrier, are used. In this particular scenario, the flow of nanoparticles is emitted by a tablet, as well depicted in Figure $8(b)$.

We assume that each nanoparticle is covered by a polymer that releases drugs, when induced by stimulation, able to cause a change in the nanostructure.

In general, stimulus-responsive polymeric nanoparticles, namely smart nanoparticles, could undergo structure, shape, and property changes after being exposed to external signals, such as $\mathrm{pH}$, temperature, magnetic field, and light, largely used to modulate the macroscopical behavior of the nanoparticles. Table 1 collects the main smart polymers used for drug delivery systems.

In the literature several works have focused on $\mathrm{pH}$ and temperature as the predominant stimulus signals, so that $\mathrm{pH}$ - and thermo-responsive nanoparticles have been extensively studied $[71,74,75,76]$. As an instance, the most commonly used thermo- and $\mathrm{pH}$-sensitive segments are poly(N-isopropy- lacrylamide) (PNIPP Am) and poly(acrylic acid) (PAA), respectively [76, 77, 78, 79].

Table 1. Examples of smart polymers used for drug delivery systems [68].

\begin{tabular}{|l|l|l|}
\hline \hline Stimulus & Polymer & Drug Released \\
\hline $\mathrm{pH}$ & $\begin{array}{l}\text { Poly(methacrylic-g-ethylene } \\
\text { glicol) - p(MMA-g-EG) }\end{array}$ & Insulin \\
\hline Electric field & $\begin{array}{l}\text { Poly(methacrylic acid)- } \\
\text { PMA }\end{array}$ & $\begin{array}{l}\text { Pilocarpine and } \\
\text { raffinose }\end{array}$ \\
\hline $\begin{array}{l}\text { Glucose con- } \\
\text { centration }\end{array}$ & $\begin{array}{l}\text { Poly(methacrylic acid-co- } \\
\text { butyl methacrylate) }\end{array}$ & Insulin \\
\hline Temperature & $\begin{array}{l}\text { Layer of Chitosan Pluronic on } \\
\text { PLGA microparticles }\end{array}$ & Indomethacin \\
\hline $\begin{array}{l}\text { Morphine con- } \\
\text { centration }\end{array}$ & $\begin{array}{l}\text { Methyl vinyl ether-Co- } \\
\text { anhydride maleic copolymer }\end{array}$ & Naltrexone \\
\hline $\begin{array}{l}\text { Urea concentra- } \\
\text { tion }\end{array}$ & $\begin{array}{l}\text { Methyl vinyl ether-Co- } \\
\text { anhydride maleic copolymer }\end{array}$ & Hydrocortisone \\
\hline \hline
\end{tabular}

\section{Diffusion process}

The characterization of the diffusion process derives from the capability to learn from biology and has mainly inspired molecular nanocommunication systems. 

ration levels.

$\frac{\partial c}{\partial t}=\frac{1}{d x}\left[J_{T}(x)-J_{T}(x+d x)\right]=-\frac{\partial J_{T}}{\partial x}$, cles in the space. expression [54]:

$J_{T}(x, t)=-D \nabla c(x, t)$ also occurs in the opposite direction. scribing the net change in this way:
In Figure 9, we can see an example of how the diffusion process works. Independently from the type of communication (i.e., molecular or electromagnetic), it is worth to discuss about the main physical principles of the fluid dynamics that are the milestones of the diffusion process. In fact, the main mechanism that drives the communication among nanoparticles is the free diffusion of nanoparti-

First of all it is useful to start from the beginning, the Fick's First Law of Diffusion. This law sums up the diffusion process through the following mathematical

where $\nabla c(x, t)$ represents the concentration gradient per unit length. It follows that $J_{T}(x, t)$ is the flow of solute, and then Eq. (10) can be identified with the nanoparticle concentration flux at the output of the transmitter, dependent on the nanoparticle concentration gradient at time $t$ and position $x$.

In practice, the Fick's First Law of Diffusion describes the diffusion as the process where a solute moves from a region of high concentration to a lower concentration area. Usually, there is a predominant direction of the flow, from the highest to the smaller concentration region, but diffusion is a complex phenomenon and

This more complex phenomenon can be described as a function of time by de-

and so, we can derive the Fick's Second Law of Diffusion as:

$\frac{\partial c}{\partial t}=D \frac{\partial^{2} c}{\partial x^{2}}$ 
Some studies present the propagation of messenger nanoparticles by means of free diffusion. In [55], the authors discuss about two simple models of pheromones diffusion in still air that are different in terms of the emitting scheme. The first one treats instantaneous emission whereas the second considers the continuous emission of pheromones. Communications via Diffusion (CvD) in a fluid are investigated in [56], where the authors show how the most of the studies about $\mathrm{CvD}$ systems is based on a very simple assumption, namely the systems are with a single transmitter and a single receiver. They analyzed a more realistic situation, that is, there are more communicating couples that make the systems much more complicated. In fact, the authors focus on the interference between closely located transmitting couples that share the same medium.

In [21] authors consider the diffusion process from a very interesting perspective, and for a very important application i.e., the development of nanorobots with sensors for nanomedicine. They show how, for an intra-body application, it is possible to apply fluid dynamics rules, by describing the fluid through the classical continuum equations. By applying the Navier-Stokes equation and the continuity condition, they are able to derive the velocity of the fluid. In their work they consider two types of forces the nanoparticles are subjected to, namely deterministic forces, due to the fluid motion, and stochastic forces due to thermal motion of nanoparticles in the fluid. Stochastic forces give rise to additional random motion, i.e., Brownian motion. The different perspective of the diffusion as considered by Cavalcanti et al. consists on the fact that a nanorobot is considered at rest with respect to the rest to the fluid, but it will be able to collect biomolecules (that is the main task of the nanorobot), due to the diffusion of the biomolecules.

Based on the main concept and the same principles of the CvD, authors in [25] show how the diffusion process works in the case of electromagnetic nanoparticles. More specifically, the authors show that gold nanoparticles can behave similarly to the molecules. In particular, different geometries are assumed, but the different nanomachines are similar in terms of volume occupancy. This characteristic simplifies the analysis of the diffusion process, even if the authors consider a three-dimensional space i.e., a lattice, to describe the diffusion phenomenon.

\section{Reception process}

In sensing applications designed for the detection of chemical and biological agents, nanoparticles (specifically, nanosensors) are usually composed of $(i)$ a recognition system or receptor, and (ii) a transduction mechanism.

Nanosensors can be based on the use of noble metal nanoparticles [57] (e.g., gold nanoparticles) and are functionalized with a biological receptor, such as an antibody, that is bound to the specific antigen of a given disease. The ligandreceptor binding [58] is, therefore, the mechanism that starts the reception process. 
1 In several works $[47,59]$, a particle receiver model is developed by taking the ligand-receptor binding mechanism into account.

The binding event between the recognition element and the target can alter physicochemical properties of the transducer that, in turn, can generate a detectable response signal. In particular, the binding creation changes the resonant frequency of the surface plasmons resulting from light irradiation. Through the LSPR technique, it is then possible to perform chemical or biological sensing.

The two main operations (i.e., binding creation, and signalling transduction) that occur in the reception process are depicted in Figure 10, for the case of sensing application through metallic nanoparticles.

From the above considerations, it follows that the reception process has the task of ( $i$ ) sensing the particle concentration at the receiver, and (ii) producing, accordingly, the output signal. As an example of sensing application, in the case of detection of BRCA1 DNA alterations [25], receptors take place only in correspondence of BRCA1 alternative splice variants, i.e., $\Delta(5 q, 6), \Delta(9,10)$, and $\Delta(11 q)$ [53].

The binding reaction occurs when the receptor was not previously bound to a particle. A chemical receptor, depending whether it is involved in a complex or not, triggers an output signal accordingly. The output signal of the end-to-end model results proportional to the rate of change in the ratio $\tau$ of the number of bound chemical receptors over the total number of chemical receptors. The trend is to reach a ratio between the number of bound chemical receptors over the total number of chemical receptors. The variation of the number of bound chemical receptors $n_{c}(t)$ inside the reception space at time $t$ is related to the number of receptor $N_{R}$ according to the following equation [49]:

$\frac{d n_{c}(t)}{d t}=N_{R} \frac{d \tau}{d t}$

According to the ligand-receptor binding reaction kinetic, when a set of nanoparticles (i.e., $N P$ ), accordingly functionalized with a given antigen, are emitted by the transmitter nanomachine, encounters with receptors (i.e., $R$ ) lying on the receiver, nanoparticles bind the receptors. These bound nanoparticle-receptors (i.e., $N P+R$ ) constitute complexes (i.e., bound receptors), as well as it is possible to release molecules $N P$ from receptors $R$, respectively according to the following chemical reactions [47],

$34(N P+R) \underset{k_{-1}}{\stackrel{k_{1}}{\rightleftarrows}} C$, 


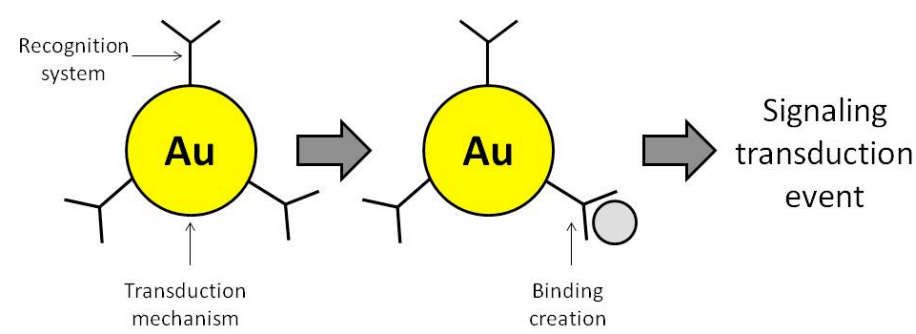

Figure 10. Schematization of the reception process in electromagnetic nanonetworks.

where $k_{1}[\mu \mathrm{mol} / \mathrm{L} / \mathrm{s}]$ and $k_{-1}\left[\mathrm{~s}^{-1}\right]$ is the rate of binding reaction, and the rate of release reaction, respectively.

In the case of drug delivery systems that make use of hollow metallic nanoparticles opportunely filled with drug concentration to be released locally, as the oral administration of a tablet for stomach disease therapy, depicted in Figure $8(b)$, the further step of drug release needs to be accomplished.

The breakage of the polymer that covers the nanoparticle is also realized by means of an electromagnetic impinging plane wave that causes the polymer chains to collapse, exposing the holes on the metallic nanoparticle, and thereby releasing the pre-loaded effector.

In [69] it was shown that for temperature increase, the core layer of PNIPAAm packed more compactly, then causing the decrease in the nanoparticle size. The drug release test showed the drug release was rapid at low $\mathrm{pH}$ medium from drugloading nanoparticle. Indeed, it is known that under a temperature stimulus, the LSPR phenomenon is established, and the free electrons of the nanoparticles follow collectively the electromagnetic oscillations. As a result, the absorbed incident electromagnetic field at the resonant wavelength is converted into heat through the photothermal effect [70]. The increase of temperature causes the polymer chains to collapse, thus exposing the holes on the nanoparticle, and thereby releasing the drug concentration. Therefore, the drug is released at the appropriate time and place.

Also, Li et al. synthesized a Y-shaped pH-/thermo-responsive copolymer P(UA-Y-NIPAAm) composed of $\mathrm{pH}$ sensitive poly(undecylenic acid) (PUA) segment and temperature-sensitive poly(N- isopropylacrylamide) (PNIPAAm) segment [72]. Soppimath et al. prepared novel temperature responsive nanoparticles with the transition at physiological temperature [73], whose structure could be deformed in acid environments to induce the release of encapsulated drug.

To summarize, the drug release mechanism based on polymers aims to [68]:

- improve the pharmaceutical profile and stability of a drug,

- ensure its correct concentration,

- achieve maximum biocompatibility,

- minimize side effects,

- stabilize the drug in vivo and in vitro,

- facilitate the accumulation of the drug at a specific site of action, 
- increase exposure time in the target cell.

The above-mentioned nanoscale sensing technologies require the use of external excitation and measurement equipment to operate. In the vision of future nanosensors devices [3] equipped with nanosensors, nanoactuator, nano-memory, nano-antenna, nano-EM transceiver, nano-processor and nano-power unit, nanodevices will be able to exchange information through nano-electromagnetic communications. By means of communication, the nanomachines will be able to accomplish more complex missions in a cooperative manner. As an example, nanosensors will be able to transmit the sensed information in a multi-hop fashion to a sink or a command centre.

For electromagnetic nanonetworks, the use of modulation and channel sharing mechanism based on the asynchronous exchange of femtosecond-long pulses, which are transmitted following an on-off keying modulation spread in time, has been proposed [12]. In [61], a receiver architecture for electromagnetic nanonetworks that make use of pulse-based modulation has been proposed. The receiver is designed in order to be simple and robust, and it is based on a Continuous-Time Moving Average (CTMA) symbol detection scheme. This scheme bases its decision in the received signal power maximum peak after the CTMA, which is implemented with a single low-pass filter. Afterwards, to decode the symbol, this maximum is compared with a previously defined threshold.

\section{Nanoparticulate Sensing and Drug Delivery}

Recently, by functionalizing the nanoparticles with biological agents such as antibodies or single stranded DNA chains, nanoparticles are forced to bind preferably to specific target cells. This aspect is the focus of extra-vivo sensing application, such as the multi-detection of DNA alterations of the BRCA1.

Several works addressing the issue of DNA detection exploit metallic nanoparticles, in order to enhance the Raman signal of fluorescent target absorbed on the metallic surface. The predominant mechanism responsible for this enhancement arises from the local electromagnetic field intensification at the surface of noble metal nanoparticles. This method is called Surface-Enhanced Raman Scattering (SERS), [80]. Among main SERS-based studies, the majority uses fluorophores as Raman labels, but it has been shown that they reduces the Raman scattering efficiency, due to the displacement of fluorophores by biological media [80].

On the other hand, techniques based on the use of metallic nanoparticles illuminated by an electromagnetic wave (visible and near infrared region) are largely exploited, as alternative approaches for gene alterations detection [62, 63]. More specifically in [25], the LSPR phenomenon for the multi-detection of BRCA1 DNA alteration, when biological gold nanoparticles are captured at the receiver, has been presented. 


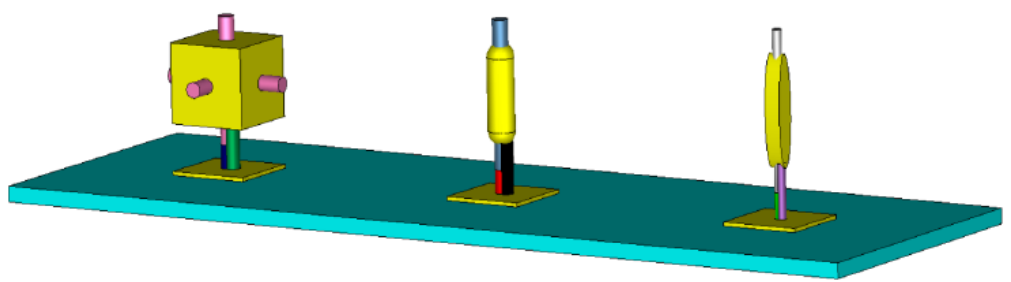

Figure 11. Ligand-receptor binding of three nanoparticles. Green, black and violet sections represent the Target Sequences for each nanoparticle [25].

In this approach, it is crucial to obtain different and independent electromagnetic responses from each nanoparticle in terms of resonant wavelength, amplitude and magnitude width. Cube, rod and elliptical cylinder nanoparticles have been functionalized with the corresponding probe sequences of alternative splicing junctions of BRCA1, as shown in Figure 11. The three corresponding DNA capture sequences of alternative splicing junctions of BRCA1 are allocated on three square gold patches of silica substrate, as depicted in Figure 11.

Furthermore, Figure 11 shows the unit cell of the sensing platform, composed of three square gold patches, functionalized with three different Capture Sequences (specifically, depicted in blue, red and green). The sensing platform is excited by an impinging plane wave, the excitation is employed to analyze the electromagnetic properties, in terms of extinction cross-section spectra, as reported in Figure 12 for the case of cube, rod, and elliptical cylinder nanoparticles, in binding and no-binding.

The meaning of this result is the lack of overlapping of the extinction crosssection spectra generated by the nanoparticles. In other words, it is crucial that the resonant peaks do not overlap, in order to obtain a multi-sensing approach. This result has been reached by using the aforementioned analytical model; in fact, they have allowed tuning the electromagnetic response for each nanoparticle. In addition to this, two cases of nanoparticle detection have been considered: the asynchronous and synchronous. In the first case, just one nanomachine has transmitted a flow of nanoparticles, and so we have just one kind of ligand-receptor bind, while in the synchronous case, all the nanomachines have transmitted the own nanoparticles, as shown in Figure 12. In this way, it is still possible to detect the DNA alteration as in the asynchronous case. The synchronous case is very important for multi-detection, since it allows the detection of different DNA alterations.

To demonstrate this ability, the extinction cross-section spectra for cube, rod and elliptical cylinder binding in the asynchronous case are shown in Figure 13 $(a),(b)$, and $(c)$, respectively. Figure 13 shows the capability of the nanosensors to distinguish the different BRCA1 alterations. Furthermore, these results prove the structure capability to be able to reveal BRCA1 alterations in the case of double binding e.g. (i) nanocube, and nanorod particle binding, (ii) nanocube and elliptical cylinder binding, and (iii) nanorod and elliptical cylinder binding. 


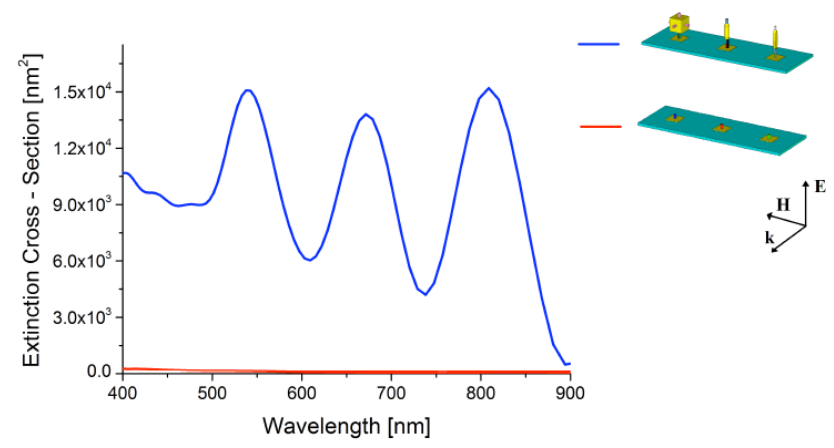

Figure 12. Synchronous nanoparticle detection [25]. Extinction Cross-Section spectra obtained when all the target sequences are bound (blue line), and are not bound (red line).

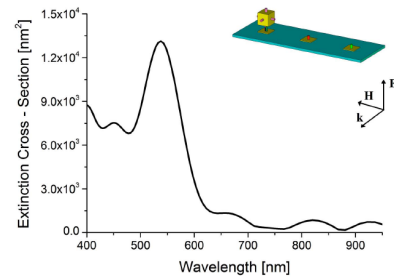

(a)

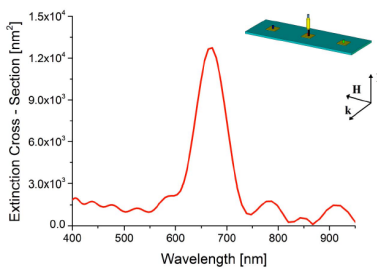

(b)

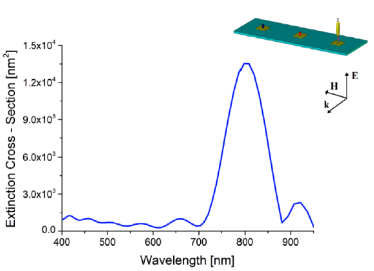

(c)

Figure 13. Asynchronous detection [25]. Extinction Cross-Section spectra obtained for binding of (a) nanocube, (b) nanorod, and (c) elliptical cylinder nanoparticle.

In the last few years several researches have focused the attention on the drug release by using functionalized gold nanoparticles [64]. The goal of this technique is to use specifics nanoparticles as drug carriers. Among the different used approaches, the photothermal effect appears as a great promise in the drug delivery field. When a metallic nanoparticle is illuminated by electromagnetic field in the visible and near infrared frequencies regime, the LSPR-induced local heating causes the release of loaded drug; this leads to enhanced drug efficacy with high spatio-temporal resolution, and very few side effects. In fact, the high electric field concentration on the nanoparticles surface ensures the heat of particle and, therefore, it causes the polymer chains to collapse, exposing the holes on the nanoparticles and thereby releasing the pre-loaded effector.

The same principle used in [25] could be efficiently applied to in-vivo drug delivery system. More specifically, it is possible to use different shapes of nanoparticles to release more types of drug. As shown in [65], there are cases that need of a multiple drug release in different times. Selective releases were induced by selective hot-spots of gold nanorods by electromagnetic field irradiation at the resonance frequency of the nanorod. In this way, at specific wavelengths it is possible to excite one type of gold nanorods, and selectively release one type of DNA strand [65]. 
By using the aforementioned nanoparticles, it is possible to load different drugs into distinct different nanoparticles shapes and by tuning the electromagnetic field frequency will be possible to excite specific nanoparticles. Therefore, by exploiting the photothermal effect, only selective drugs will be released, in the case of drug delivery applications and only selective DNA chains will be detected, in the case of sensitive applications. As results, the use of different nanoparticles shapes enables independent control over the release of each drug by tuning the nanoparticles, leading to a programmed release of multiple drugs.

Finally, another work dealing on the use of nanoparticles for sensing applications (i.e., detection of DNA alterations) is given in [81]. Specifically, two-layers nanostructures are considered, comprising of a $\mathrm{SiO} 2$ core, and a gold shell with different thicknesses. By varying the ratio of the core/shell radius, it is possible to tune the electromagnetic response. Indeed, the optical cross-section propriety obtained for nanoshells with different radii is higher than that one obtained for the nanoshells with the same volumes.

Simulation results in [81] show the extinction cross-section spectra in the case of (i) synchronous nanoparticle transmission, (ii) asynchronous nanoparticle transmission, and (iii) no nanoparticle reception. The extinction cross-section spectra show a peak at $550 \mathrm{~nm}$ for both synchronous and asynchronous transmission, but the magnitude of the spectral signal is higher for the synchronous transmission, with respect to the asynchronous case. In both two cases, no shift of the resonant wavelength occurs. From a physical point of view, this means that there are no coupling effects among nanoparticles. Finally, as expected, in the case of no nanoparticle reception, the extinction cross-section spectrum is approximately zero.

\section{Conclusions}

In this chapter we have addressed recent advances in electromagnetic nanonetworks for biomedical applications. Starting from the description of most used nanoparticles for sensing and drug delivery systems (i.e., nanorods, nanocubes, bowtie nanoparticles, etc.), we have presented the analytical models behind their specific properties. By exploiting the well-known LSPR phenomenon, electromagnetic nanoparticles are shown to be very suitable for sensing (i.e., analysis of concentration of specific substances), and drug delivery applications (i.e., nanoparticles are intended as drug carriers for localized drug release).

Furthermore, under the assumption of similarity of electromagnetic nanoparticles with biological molecules (i.e., small size of the structure), we rely on specific laws in the nanoscale. The channel model linking one transmitter to one receiver is generally represented by a liquid environment (i.e., the human blood flood or the biological tissue), and is subjected to specific processes (i.e., nanoparticle trans- 
mission, diffusion, and reception). Once nanoparticles are captured by the receptors, the detection process occurs by means of LSPR phenomenon.

Specific sensing and drug delivery applications of nanoparticles are also presented, regarding the detection of DNA alterations of BRCA1 gene, as well as for fighting stomach diseases. The case of different shapes of nanoparticles has been addressed, both for sensing and drug delivery applications, by showing how different geometric forms can be exploited for multi-detection sensing and to effectively regulate programmed drug delivery, respectively.

Simulation results assess the validity of the use of electromagnetic nanoparticles for these biomedical applications.

As a future investigation, novel nanomaterials are promising for the design of innovative nanodevices. As an instance, graphene-based nanoantennas can provide outstanding sensing capabilities, as well as graphene-based transistors are not only smaller, but predictably faster too.

\section{References}

[1] R. A. Freitas Jr., "What is nanomedicine?," Nanomedicine: Nanotechnology, Biology and Medicine, Vol. 1, No. 1, pp. 2-9, March 2005.

[2] R. A. Freitas Jr., Nanomedicine, Volume I: Basic Capabilities, Landes Bioscience, Georgetown, TX, 1999.

[3] I. F. Akyildiz and J. M. Jornet, Electromagnetic Wireless Nanosensor Networks, Nano Communication Networks, Vol. 1, pp. 3-19, March 2010.

[4] R. A. Freitas Jr., "Current Status of Nanomedicine and Medical Nanorobotics," in Journal of Computational and Theoretical Nanoscience, Vol. 2, pp. 1-25, 2005.

[5] I. F. Akyildiz, J. M. Jornet, and M. Pierobon, "Nanonetworks: A New Frontier in Communications," Communications of the ACM, Vol. 54, No. 11, pp. 84-89, 2011.

[6] I. F. Akyildiz, F. Brunetti, and C. Blzquez, Nanonetworks: a New Communication Paradigm, Computer Networks, Vol. 52, No. 12, pp. 2260-2279, 2008.

[7] J.M. Jornet and I.F. Akyildiz, Channel Capacity of Electromagnetic Nanonetworks in the Terahertz Band, in Proc. of the IEEE International Conference on Communications, ICC 2010, Cape Town, South Africa, May 2010.

[8] X. Wang, X. Lou, Y. Wang, Q. Guo, Z. Fang, X. Zhong, H. Mao, Q. Jin, L. Wu, H. Zhao, and J. Zhao, "QDs-DNA Nanosensor for the Detection of Hepatitis B Virus DNA and the Single-base Mutants," Biosens Bioelectron., Vol. 25, No. 8, pp. 1934-1940, 2010.

[9] A. Abraham, R. Kannangai, and G. Sridharan, "Nanotechnology: A New Frontier in Virus Detection in Clinical Practice," Indian Journal of Medical Microbiology, Vol. 26, No. 4, pp. 297-301, 2008.

[10] I. F. Akyildiz, F. Fekri, R. Sivakumar, C. R. Forest, and B. R. Hammer, "Monaco: Fundamentals of molecular nano-communication networks," IEEE Wireless Communications, Vol. 19, No. 5, pp. 12-18, 2012.

[11] J. M. Jornet and I. F. Akyildiz, "Channel modeling and capacity analysis for electromagnetic wireless nanonetworks in the terahertz band," IEEE Transactions on Wireless Communications, Vol. 10, No. 10, pp. 3211-3221, 2011.

[12] J. M. Jornet, and I. F. Akyildiz, "Low-Weight Channel Coding for Interference Mitigation in Electromagnetic Nanonetworks in the Terahertz Band," in Proc. of the IEEE International Conference on Communications (ICC 2011), June 5-9, Kyoto, Japan. 
[13] J. M. Jornet, and I. F. Akyildiz, "PHALME: A Physical Layer Aware MAC Protocol for Electromagnetic Nanonetworks," in Proc. of the IEEE International Conference on Communications (ICC 2011), June 5-9, Kyoto, Japan.

[14] J. M. Jornet, and I. F. Akyildiz, "Joint Energy Harvesting and Communication Analysis for Perpetual Wireless Nanosensor Networks in the Terahertz Band," IEEE Transactions on Wireless Communications, Vol. 11, No. 3, pp. 570-580, 2012.

[15] S. Svenson and R. K. Prud'homme, "Multifunctional Nanoparticles for Drug Delivery Applications: Imaging, Targeting, and Delivery," Springer, Ed., 2012.

[16] A. Swami, J. Shi, S. Gadde, A. Votruba, N. Kolishetti, and O. Farokhzad, "Nanoparticles for targeted and temporally controlled drug delivery," in Multifunctional Nanoparticles for Drug Delivery Applications: Imaging, Targeting, and Delivery, S. Svenson and R. Prud'homme, Eds. Springer, 2012.

[17] Y. Zhou, Y. Kong, S. Kundu, J. Cirillo, and H. Liang, "Antibacterial activities of gold and silver nanoparticles against escherichia coli and bacillus calmette-gurin," Journal of Nanobiotechnology, Vol. 10, 2012.

[18] M. Hossen, K. Kajimoto, H. Akita, M. Hyodo, and H. Harashima, "Vascular-targeted nanotherapy for obesity: Unexpected passive targeting mechanism to obese fat for the enhancement of active drug delivery," J. Control. Release, Vol. 163, pp. 101-110, 2012.

[19] D. Vllasaliu, C. Alexander, M. Garnett, M. Eaton, and S. Stolnik, "Fc-mediated transport of nanoparticles across airway epithelial cell layers," J. Control. Release, Vol. 158, pp. 479486, 2012.

[20] D. Bhumkar, H. Joshi, M. Sastry, and V. Pokharkar, "Chitosan reduced gold nanoparticles as novel carriers for transmucosal delivery of insulin," Pharmaceutical Research, Vol. 24, 2007.

[21] A. Cavalcanti, B. Shirinzade, R. A. Freitas, and T. Hogg, "Nanorobot Architecture for Medical Target Identification," Nanotechnology, vol. 19, no. 1, 2008.

[22] Y. Chahibi, M. Pierobon, S. Song, and I. Akyildiz, "A Molecular Communication System Model for Particulate Drug Delivery Systems," IEEE Transactions on Biomedical Engineering, June 2013.

[23] T. Nakano, M. J. Moore, Y. Okaie, A. Enomoto, and T. Suda, "Swarming Biological Nanomachines Through Molecular Communication for Targeted Drug Delivery," in Proc. of IEEE Conference on Soft Computing and Intelligent Systems and Symposium on Advanced Intelligent Systems, November 2012.

[24] V. Loscrì, E. Natalizio, V. Mannara, and G. Aloi, "A Novel Communication Technique for Nanobots based on Acoustic Signals," in Proc. of the 7th International Conference on BioInspired Models of Network, Information, and Computing Systems, ser. Bionetics'12, Lugano, Switzerland, 2012.

[25] R. Iovine, V. Loscrì, S. Pizzi, R. Tarparelli, and A. M. Vegni, "Model of Multi-Source Nanonetworks for the Detection of BRCA1 DNA Alterations Based on LSPR Phenomenon," Advances in Nanoparticles, Vol. 2, No. 4, pp. 301-312, 2013.

[26] L. Dykman, and N. Khlebtsov, "Gold nanoparticles in biomedical applications: recent advances and perspectives," Chem. Soc. Rev., Vol. 41, No. 6, pp. 2256-2282, 2012.

[27] A. Kumar, B. M. Boruah, and X.-J. Ling, "Gold Nanoparticles: Promising Nanomaterials for the Diagnosis of Cancer and HIV/AIDS," Journal of Nanomaterials, Vol. 2011, pp. 1-17, 2011.

[28] C. R. Patra, R. Bhattacharya, D. Mukhopadhyay, and P. Mukherjee, "Fabrication of Gold Nanoparticles for targeted therapy in pancreatic cancer," Advanced Drug Delivery Reviews, Vol. 62, No. 3, pp. 346-361, 2010.

[29] E. C. Cho, C. Glaus, J. Chen, M. J. Welch, and X. Xia, "Inorganic nanoparticle-based contrast agents for molecular imaging," Trends. Mol. Med., Vol .16, No. 12, pp. 561-573, 2010.

[30] W. Cai, T. Gao, H. Hong, and J. Sun, "Applications of gold nanoparticles in cancer nanotechnology," Nanotechonology, Vol. 2008, No. 1, pp. 17-32, 2008. 
[31] Z. Salamon, H.A. Macleod and G. Tollin, "Surface plasmon resonance spectroscopy as a tool for investigating the biochemical and biophysical properties of membrane protein systems. I: Theoretical principles," Biochimica and Biophysica Acta, Vol. 1331, No. 2, pp. 117$129,1997$.

[32] L. B. Sagle, L. K. Ruvuna, J. A. Ruemmele, and R. P. Van Duyne, "Advances in localized surface plasmon resonance spectroscopy biosensing," Nanomedicine, Vol. 6, No. 8, pp. 1447$1462,2011$.

[33] A. Moores, and F. Goettmann, "The plasmon band in noble metal nanoparticles: an introduction to theory and applications," New Journal of Chemistry, Vol. 30, pp. 1121-1132, 2006.

[34] J. G. Van Bladel, “Electromagnetic Fields,” John Wiley \& Sons, Hoboken, 2007.

[35] L. La Spada, R. Iovine, and L. Vegni, "Nanoparticle Electromagnetic Properties for Sensing Applications," Advances in Nanoparticles, Vol. 1, pp. 9-14, 2012.

[36] R. Iovine, L. La Spada, and L. Vegni, "Nanoparticle device for biomedical and optoelectronics applications," COMPEL, Vol. 32, No. 5, pp. 1596-1608, 2013.

[37] X. Xu, Y. Ying, and Y. Li, "Gold Nanorods Based LSPR Biosensor for Label-Free Detection of Alpha-Fetoprotein,” Procedia Engineering, Vol. 25, pp. 67-70, 2011.

[38] L. La Spada, R. Iovine, and L. Vegni, "Electromagnetic modeling of ellipsoidal nanoparticles for sensing applications," Optical Engineering, Vol. 52, No. 5, pp. 1-5, 2013.

[39] R. Iovine, L. La Spada, and L. Vegni, "Optical Properties of Modified Nanorod Particles for Biomedical Sensing," IEEE Transactions on Magnetics, Vol. 50, No. 2, to appear, 2014.

[40] A. Tanaka, and B. Nakamura, "Optical Imaging: Technology, Methods and Applications," Nova Science Publisher, 2012.

[41] R. Iovine, L. La Spada, and L. Vegni, "Modified Bow-Tie Nanoparticles Operating in the Visible and Near Infrared Frequency Regime," Advances in Nanoparticles, Vol. 2, No. 1, pp. 21-27, 2013.

[42] T. Suda, M. Moore, T. Nakano, R. Egashira, and A. Enomoto, "Exploratory research on molecular communication between nanomachines," in Proc. of Genetic and Evolutionary Computation Conference, (GECCO'05), ACM, 2005.

[43] A. Einolghozati, M. Sardari, A. Beirami and F. Fekri, "Capacity of Discrete Molecular Diffusion Channels," in Proc. of International Symposium on Information Theory (ISIT 2011), Saint Petersburg, Russia, July 2011.

[44] A. Keramidas, A. J. Moorhouse, P. R. Schofield, and P. H. Barry, "Ligand-gated ion channels: mechanisms underlying ion selectivity," Progress in Biophysics and Molecular Biology, vol. 86, no. 2, pp. 161- 204, 2004.

[45] M. A. Model and G. M. Omann, "Ligand-receptor interaction rates in the presence of convective mass transport," Biophysical Journal, Vol. 69, No. 5, pp. 1712-1720, 1995.

[46] B. Atakan, and O.B. Akan, "On Channel Capacity and Error Compensation in Molecular Communication,” Transactions on Computational Systems Biology X (2008), pp. 59-80.

[47] B. Atakan, and O.B. Akan, "An Information Theoretical Approach for Molecular Communication," in Proc. of 2nd Bio-Inspired Models of Network, Information and Computing Systems, Bionetics 2007, pp. 33-40, 10-12 Dec. 2007.

[48] B. Atakan, and O.B. Akan, "Deterministic capacity of information flow in molecular nanonetworks," Nano Communication Networks (Elsevier), Vol. 1, No. 1, 2010, pp. 31-42.

[49] M. Pierobon and I. Akyildiz, "A physical end-to-end model for molecular communication in nanonetworks," IEEE Journal on Selected Areas in Communications, Vol. 28, No. 4, pp. 602-611, 2010.

[50] T. Nakano, Y. Okaie, and J-Q. Liu,, "Channel model and capacity analysis of molecular communication with brownian motion," IEEE Communications Letters, Vol. 16, No. 6, June 2012.

[51] I. Llatser, E. Alarcòn, and M. Pierobon, "Diffusion-based Channel Characterization in Molecular Nanonetworks," in Proc. of IEEE Conference on Computer Communications Workshops (INFOCOM WKSHPS), pp. 467-472, 10-15 April 2011. 
[52] L. Kadouri, A. Hubert, Y. Rotenberg, T. Hamburger, M. Sagi, C. Nechushtan, D. Abeliovich and T. Peretz, "Cancer Risks in Carriers of the BRCA1/2 Ashkenazi Founder Mutations," Journal of Medical Genetics, Vol. 44, No. 7, pp. 467-471, 2007.

[53] D. Thompson, D. Easton, and B. C. L. Consortium, "Cancer incidence in BRCA1 mutation carriers," Journal of the National Cancer Institute, Vol. 94, No. 18, p. 1358-1365, 2002.

[54] L. Sun, C. Yu, and J. Irudayaraj, "Raman multiplexers for alternative gene splicing," Analytical Chemistry, Vol. 80, No. 9, pp. 3342-3349, 2008.

[55] E. L. Cussler, "Diffusion. Mass Transfer in Fluid Systems," 2nd edition, Cambridge University Press, 1997.

[56] L. Parcerisa, and I.F. Akyildiz, "Molecular communication options for long range nanonetworks," Computer Networks Journal 53, 16 (Nov. 2009), Elsevier, 2753-2766.

[57] Mehmet Şükrü Kuran' H. Birkan Yilmaz, T. Tugcu, I. F. Akyildiz "Interference effects on modulation techniques in diffusion based nanonetworks," in Elsevier Nano Communication Networks, Vol. 3, No. 1, March 2012, pp. 65-73.

[58] K. Saha, S. S. Agasti, C. Kim, X. Li, and V. M. Rotello, "Gold Nanoparticles in Chemical and Biological Sensing," Chemical Reviews 2012, 112(5), pp. 2739-2779.

[59] J.-P. Rospars, V. Krivan, and P. Lansky, "Perireceptor and Receptor Events in Olfaction. Comparison of Concentration and Flux Detectors: a Modeling Study," Chemical Senses, Vol. 25, pp. 293-311, 2000.

[60] B. Atakan and O. B. Akan, "On molecular multiple-access, broadcast, and relay channels in nanonetworks," in Proc. of the ICST/ACM Conference BIONETICS 2008, Japan, November $25-28,2008$

[61] R. G. Cid-Fuentes, J. M. Jornet, I. F. Akyildiz and E. Alarcon, "Receiver Architecture for Pulse-based Electromagnetic Nanonetworks in the Terahertz Band," in Proc. of International Conference on Communications, Ottawa, Canada, June 10-15, 2012.

[62] W. Zhou, Y. Ma, H. Yang, Y. Ding, and X. Luo, "A label-free biosensor based on silver nanoparticles array for clinical detection of serum p53 in head and neck squamous cell carcinoma," International Journal of Nanomedicine, Vol. 2011, No. 6, pp. 381-386, 2011.

[63] Y. N. Tan, X. Su, Y. Zhu, and J. Y. Lee, "Sensing of Transcription Factor through Controlled-Assembly of Metal Nanoparticles Modified with Segmented DNA Elements," ACS Nano, Vol. 4, No. 9, pp. 5101-5110, 2010.

[64] S. Rana, A. Bajaj, R. Mout, and V. M. Rotello, "Monolayer coated gold nanoparticles for delivery applications," Advanced Drug Delivery Reviews, Vol. 64, No. 2, pp. 200-216, 2012.

[65] A. Wijaya, S. B. Schaffer, I. G. Pallares, and K. Hamad-Schifferli, "Selective Release of Multiple DNA Oligonucleotides from Gold Nanorods," ACS Nano, Vol. 3, No. 1, pp. 80-86, 2009.

[66] S. Redner, A guide to first-passage processes. Cambridge University Press, 2001.

[67] H. B. Yilmaz, N-R. Kim, and C-B. Chae, "Effect of ISI Mitigation on Modulation Techniques in Communication via Diffusion," in Proc. of $1^{s t}$ ACM International Conference on Nanoscale Computing and Communication, Atlanta, May 13-14, 2014.

[68] G. Vilar, J. Tulla-Puche, and F. Albericio, "Polymers and Drug Delivery Systems," in Current Drug Delivery, 2012, Vol. 9, No. 4, pp. 367-394.

[69] S.W. Hong, D.Y. Kim, J.U. Lee, and W.H. Jo, "Synthesis of Polymeric Temperature Sensor Based on Photophysical Property of Fullerene and Thermal Sensitivity of Poly(Nisopropylacrylamide)," in Macromolecules, Vol. 42, pp. 2756-2761, February, 2009.

[70] J.-H. Lee, J.-T Jang, J.-T. Jang, J.-S. Choi, S. H. Moon, S.-H. Noh, J.-W. Kim, J.-G. Kim, K.I. Park, and J. Cheon, "Exchange-coupled Magnetic Nanoparticles for Efficient Heat Induction," in Nature Nanotechnology Letter, Vol. 6, pp. 418-422, June 2011.

[71] Y.Y. Li, H. Q. Dong, K. Wang, and D.L. Shi, X. Z. Zhang, and R.X. Zhuo, "Stimulusresponsive polymeric nanoparticles for biomedical applications," in Science China Chemistry, Vol. 53, No. 3, pp. 447-457, 2010. 
[72] Y.Y. Li, X.Z. Zhang, H. Cheng, G.C. Kim, S.X. Cheng, and R.X. Zhuo, "Novel stimuliresponsive micelle self-assembled from Y -shaped P(UA-Y - NIPAAm) copolymer for drug delivery," in Biomacromolecules, 2006, Vol. 7, No. 11, pp. 2956-2960.

[73] K.S. Soppimath, D.C.W. Tan, and Y.Y. Yang, "PH-triggered thermally responsive polymer core-shell nanoparticles for drug delivery", Adv. Mater, 2005, Vol. 17, No. 3, pp. 318-323.

[74] D. Schmaljohann, "Thermo- and $\mathrm{pH}$-responsive polymers in drug delivery," Adv. Drug Deliver Rev, 2006, Vol. 58, No. 15, pp. 1655-1670.

[75] N. Suchaoin, S. Chirachanchai, and S. Perrier, "PH- and thermo-multi-responsive fluorescent micelles from block copolymers via reversible addition fragmentation chain transfer (RAFT) polymerization,” Polymer, 2009, Vol. 50, No. 17, pp. 4151-4158.

[76] Q.S. Zhang, L.S. Zha, J.H. Ma, and B.R. Liang, "A novel route to prepare pH- and temperature-sensitive nanogels via a semibatch process," J. Colloid Interf Sci, 2009, Vol. 330, No. 2, pp. 330-336.

[77] S. Ganta, H. Devalapally, A. Shahiwala, and M. Amiji, "A review of stimuli-responsive nanocarriers for drug and gene delivery," J. Control Release, 2008, Vol. 126, No. 3, pp. 187204.

[78] C. Alexander, and K.M. Shakesheff, "Responsive polymers at the biology/ materials science interface," Adv Mater, 2006, Vol. 18, No. 24, pp. 3321-3328.

[79] N. Rapoport, "Physical stimuli-responsive polymeric micelles for anti-cancer drug delivery," Prog Polym Sci, 2007, Vol. 32, No. 8-9, pp. 962-990.

[80] F. T. Docherty, M. Clark, G. McNay, D. Graham, and W.E. Smith, "Multiple labelled nanoparticles for bio detection," Faraday Discuss., Vol. 126, pp. 281-288, October 2003.

[81] R. Iovine, R. Tarparelli, and A.M. Vegni, "Detection of DNA alterations using Gold Nanoparticles exploiting the LSP phenomenon," in Proc. of 21st IEEE Intl. Conf. on Applied Electromagnetics and Communications (ICECOM), October 14-16, 2013, Dubrovnick, Croatia. 